\title{
Impact of Variable Bit Rate and Packet Size on the Performance Evaluation of Neighbor Aware AODV and DSDV Routing Protocols for MANET's
}

\author{
E. Gnanamanoharan \\ Assistant Professor \\ Dept. of Electrical Engg., \\ Annamalai University, \\ Annamalainagar - 608002 . \\ Tamilnadu, India.
}

\author{
R.Bensraj \\ Assistant Professor \\ Dept. of Electrical Engg., \\ Annamalai University, \\ Annamalainagar - 608002 . \\ Tamilnadu, India.
}

\begin{abstract}
Mobile Ad hoc Networks (MANET) have become an exciting and important technology in recent years because of the rapid proliferation of wireless nodes. They consist of wireless host that can move freely, rapidly changing, which are likely composed of relatively limited bandwidth and low powered wireless links. MANET is a wireless mobile network in which mobile nodes can transmit and receive without any fixed infrastructure and can be distributed for many applications such as soldiers relaying critical information, business associates sharing information during get together. Authentic and efficient routing is significant and vital in those benefits. A major dispute that lies in MANET communication is the limit less mobility and more frequent link failures. Conventional routing algorithms are therefore deficient for ad-hoc networks. In this paper we propose a modified approach based on the AODV [2] which affords more beneficial results when compared with the conventional AODV and DSDV protocols. Simulations are accomplished in ns-2 and various performance metrics like throughput, end to end delay, Packet loss and pdr $[5,6,8]$ with variable bit rate, packet size and node density and pause time are computed.
\end{abstract}

\section{Keywords}

MANET Routing, AODV, DSDV, NS2, Metrics

\section{INTRODUCTION}

In MANET, wireless nodes acts as a central coordinator to store the incoming packets and forward it to the destination. They also move randomly, independent of one another, irrespective of topology which makes routing much unmanageable. Sending nodes in a MANET usually seek the help of other neighboring nodes to set up routes to reach destination nodes. A number of routing protocols are addressed in many literatures. Compared to table driven protocols, on demand routing protocols can do effort less routing overhead. The probability based, counter based, area based, location based and locality based approaches have been suggested to carry off the effect of flooding.

This paper is presented as follows: Section 1, deals a brief review of broadcasting in MANET Section 2, we have discussed the features of the AODV, DSDV and Neighbor Aware AODV protocol,Section3,we have presented simulation and performance metrics. Section 4, the performance of AODV, DSDV and Neighbor Aware AODV.

\section{MANET ROUTING PROTOCOLS}

\subsection{Ad hoc On Demand Distance Vector (AODV)}

AODV is a on demand routing protocol and works in two stages they are route discovery and maintenance. Route discovery is the main features of MANET protocol in wireless environment. AODV discover the required route discovery by sending RREQ to entire neighbors. Redundant packets are ignored by the sequence numbers. When RREQ packet comes at a host receives a present route and it finds whether the route is current by checking the destination sequence numbers in its route list to the destination sequence number in the route request packet. If the RREQ's sequence number for the destination is greater than that of new one the intermediate node wouldn't send reply to the sender.

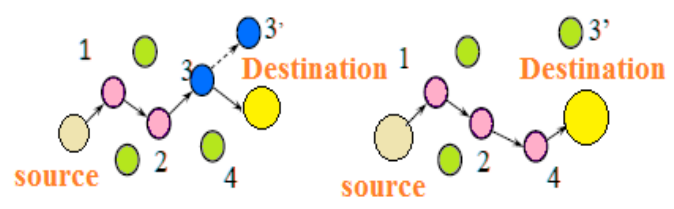

Figure 1: Route Maintenance in Mobile Ad hoc Network

The route maintenance serves to maintain the routes while there is link broken or node failure and hidden nodes. Such rout failures are displayed by Route Error packets. When a wireless node gets an Route error, the route is not valid and entering the distance to the receiving node is infinity in the route list. Once again need to start discovering of new route. In on demand routing protocol, when a sender needs to communicate, it has to finds a suitable route and utilize it during the packet transmission and it should maintain this route till the packet sending is getting over.

\subsection{Destination Sequence Distance Vector (DSDV)}

DSDV is one of table driven protocol based on Bellman-Ford algorithms requiring every node to periodically broadcast the current route information and updates it. It works every node as a specialized central coordinator which periodically update's its knowledge of the network with the neighbor nodes in the network. The routing table comprises a sequence number and it is even if there is link else is marked as odd. By chance all the new updates have the same sequence number, 
the smallest is selected and the representing previous entry in the table is considered as a less priority route and discarded and control packet with this sequence number is sent to the entire nodes. Routing information is distributed between nodes by sending full dumps randomly and smaller incremental updates more frequently. Whenever frequent changes in the topology, need to use a new sequence number. Due to this reason, DSDV is not preferred for dynamic networks.

\subsection{Neighbor Aware Ad hoc On-Demand Distance Vector (NAAODV)}

In the literature survey, the overall network performance affected by increased routing overhead delay and reduced throughput due to unsighted flooding of redundant RREQ packets to entire nodes[2]. The flooding is a major concern in route request phase in AODV. The new approach NAAODV discovers in a first step, all possible routes to all nodes.. We use the neighbor aware information of the region to find out node density to maintain the obtained route. If the network is dense, no need to rebroadcast of RREQ packets to nearby nodes which will be more beneficial to reduce overhead. Intermediate node is considered to be located at a dense region of the network if its number of neighbors is more than the average number of neighbors $n$ in the network[1].Here the data dissemination concept is based on the priority and it is set to high or low based on information regarding location the location of neighbor and energy information whether the neighbor is adequate energy for effective routing.

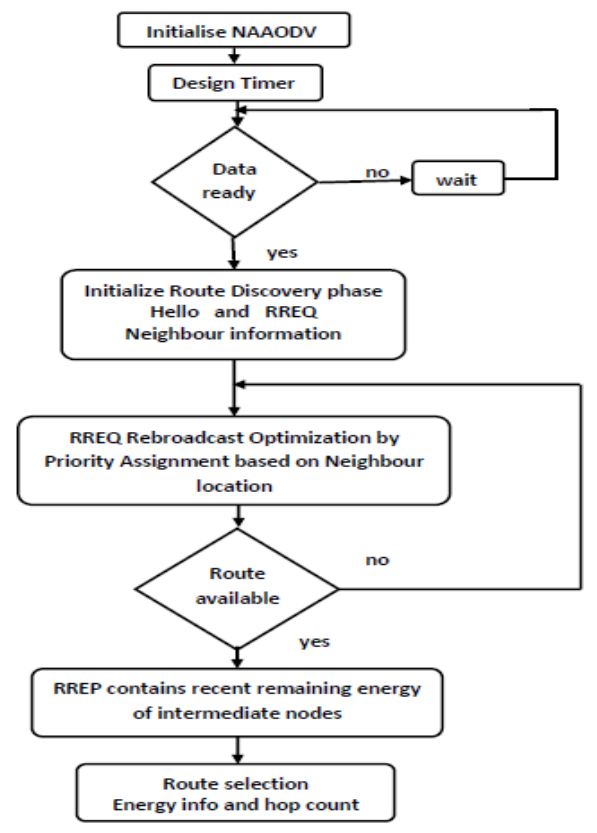

Figure 2: Flow chart of NAAODV

The priority is high when the neighbor node is located far away from source node else set to low when it is locate near to it. So we could avoid unnecessary retransmission. Also need to check the neighbor presence and a host has sufficient neighbors to destroy channel contention and collision and could the network performance [2].Thus the optimization of rebroadcast of RREQ is based on composite metric of priority assignment at RREQ packet and most recent remaining energy information in the RREP packet. Its evaluated by measurement of energy.

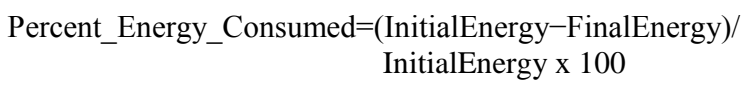

Average_Energy_Consumed=Sum_of_Percent_Energy_

Consumed_by_All_Nodes / Number_of_Nodes

when calculating the energy consumed to transmit a packet $p$ is $\mathrm{E}(\mathrm{p})=\mathrm{i} * \mathrm{v} * \mathrm{tp} * \mathrm{ps}$ Joules are needed. Here, $\mathrm{i}$ is the current, $\mathrm{v}$ is the voltage and tp is the time taken to transmit the packet $p$ and $\mathrm{ps}$ is the size of packet. Once RREP is received from destination, need to choose the best route based on latest energy information of intermediate nodes using routing table update. We modified the source code of AODV in Ns 2-2.34 to implement this proposed approach as shown in Fig.2. Additional fields are appended to RREP to persuade the energy information(see Figure 2).

\section{PROTOCOL IMPLEMENTATION AND SIMULATION ENVIRONMENT}

NS2 is an open-source event-driven simulator developed particularly for simulation of data networks. It is based on two languages written in $\mathrm{C}++$ and OTCL used to execute researcher's script. Network Simulator 2, the most used network simulator provides an easy way to fulfill this exhaustive task allowing users to trace packet flow, filter packets, visualize nodes position and movements and so on. To evaluate network performance, can configure network components, visualize the traffic flow by NAM and accumulate and display the results by Xgraph using TCL script. The Network Animator (NAM) can be used to visualize packets, the parsing of the NS-2 trace file using AWK scripts developed by users for the requires a further step to do data processing and graphical organization and able to easily translate the NS-2 trace files into graphics and to give a summary of what occurred during simulations, has led to the development of a new data analysis.

\subsection{Simulation Setup}

We utilized the NS2 network simulator (version 2.34) to conduct extensive experiments to study the behavior of the proposed Neighbor aware AODV.

Table 1. simulation parameters

\begin{tabular}{|c|c|}
\hline Parameter & Value \\
\hline Coverage Area & $1500 \mathrm{~m}$ x 1500m \\
\hline Channel & Wireless channel \\
\hline Radio Propagation & Two Ray Ground \\
\hline Antenna type & Omni directional antenna \\
\hline Transmission Range & $250 \mathrm{~m}$ \\
\hline MAC layer protocol & IEEE 802.11 \\
\hline Number of Nodes & $10,30,50$ \\
\hline Mobility Model & Random Waypoint \\
\hline Bit Rate & $100,500,1000 \mathrm{~kb}$ \\
\hline Packet Size & $256,512,1024$ bytes \\
\hline Routing Protocol & NAAODV, AODV, DSDV \\
\hline Simulator & NS2 (2.34) \\
\hline
\end{tabular}

Various set of mobile hosts $(10,30$ and 50$)$ are randomly distributed in a simulation area of $1500 \mathrm{~m} \mathrm{X} 1500 \mathrm{~m}$ with random waypoint model is considered for evaluation. A 
comprehensive set of parameters of packet delay, packet loss, throughput and Packet delivery ratio, through variable packet size 256,512 and 1024 bytes, bit rates $100,500,1000 \mathrm{~kb}$. Table 1 shows the parameter and Fig.3 shows a snapshot of the simulation process conducted. Figure 4 represents the sequence of implementation for comparison.

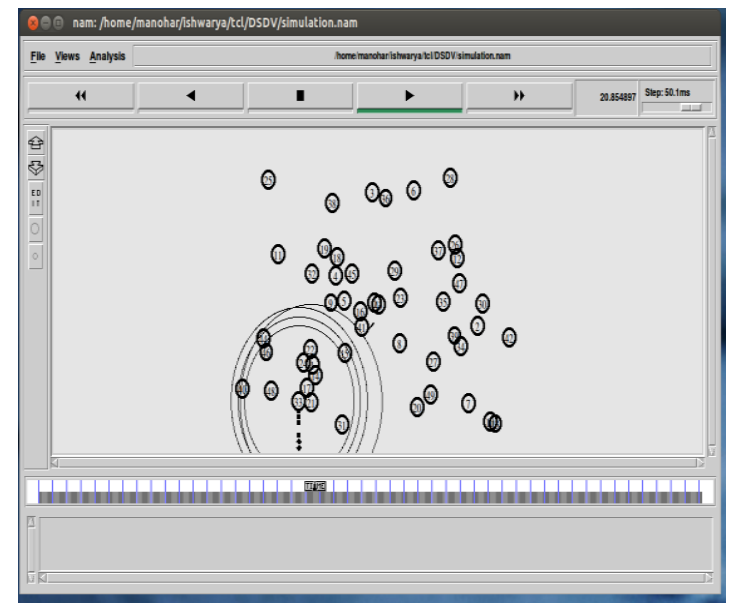

Figure 3: Wireless connections among the nodes

The trace files are further analyzed using number of AWK scripts and results are displayed using $\mathrm{x}$ graph utility. The sample script is shown in Figure 5.

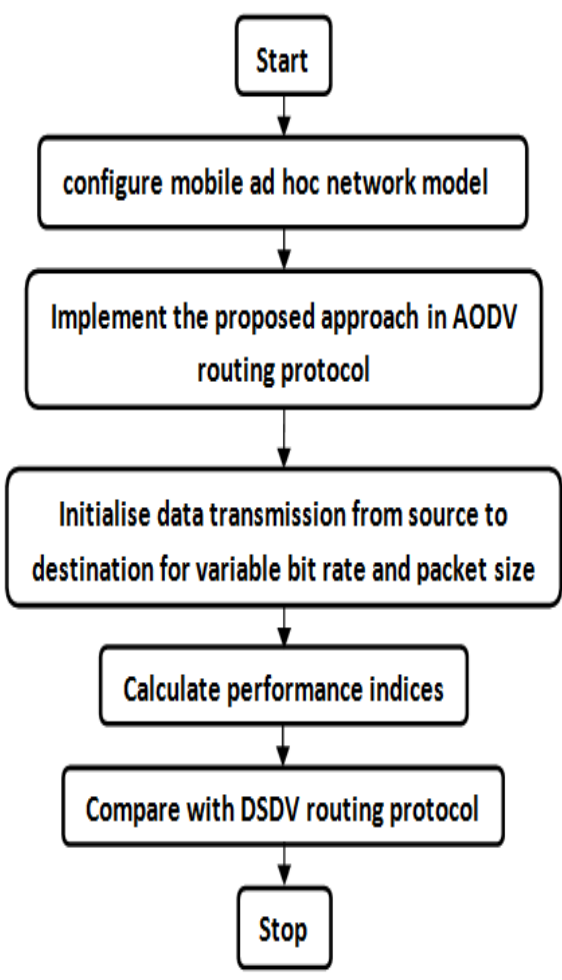

Figure 4: Sequence of Implementation

\subsection{Performance Indices}

The following performance indices are computed.

\subsubsection{Network Throughput}

It is a measurement of total data packets by the receiver.

\subsubsection{Packet loss}

Total number of data packets dropped by receiver.

\subsubsection{End to End Delay}

The time taken for transmission of data packets from sending send to receiving end.

\subsubsection{Packet delivery ratio}

It is defined as the percentage of data packets obtained by the destination node. It shows the ability and routing effectiveness of routing protocol in wireless network.

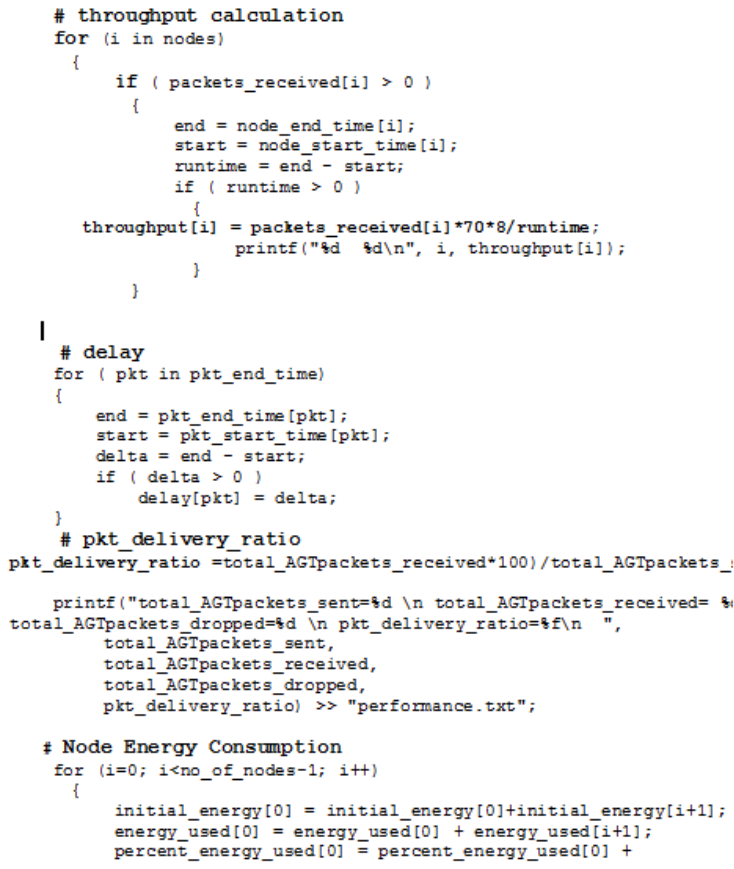

Figure 5: Sample AWK script for Metric Evaluation

\section{RESULTS AND DISCUSSION}

4.1 Impact of variable Bit rate and packet size at Network throughput

The first important measure of throughput is analyzed with variation in packet size and bit rate with respect to pause time. When increasing bit rate from 100 to 500, It has been identified that the throughput of NAAODV is increases except $1000 \mathrm{kbps}$ as shown in Figure 4. NAAODV improves a increased throughput compare with AODV and DSDV and also NAAODV outperform compare with AODV and DSDV when increased packet size at $256 \mathrm{~b}$ and $512 \mathrm{~b}$ but the performance of NAAODV degrades as network load is increased to 1024 bytes as shown in Figure 7. 


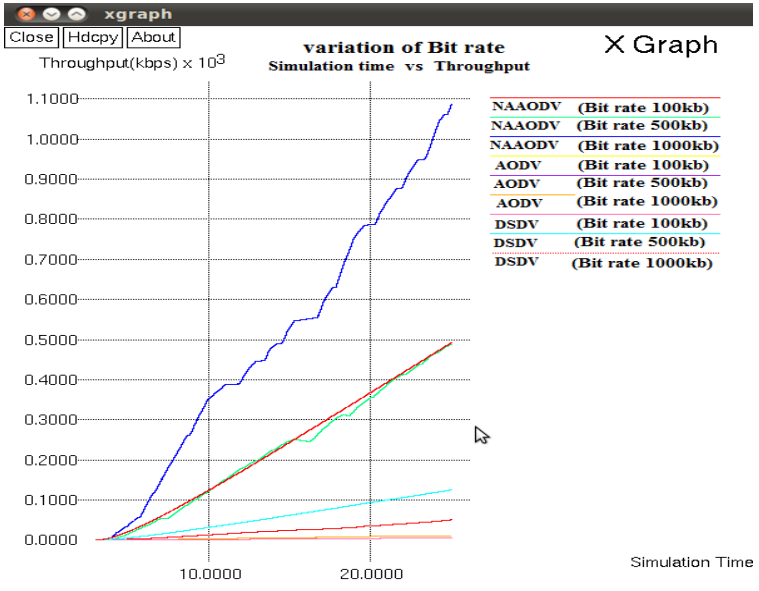

Figure 6: Throughput at variable bit rate

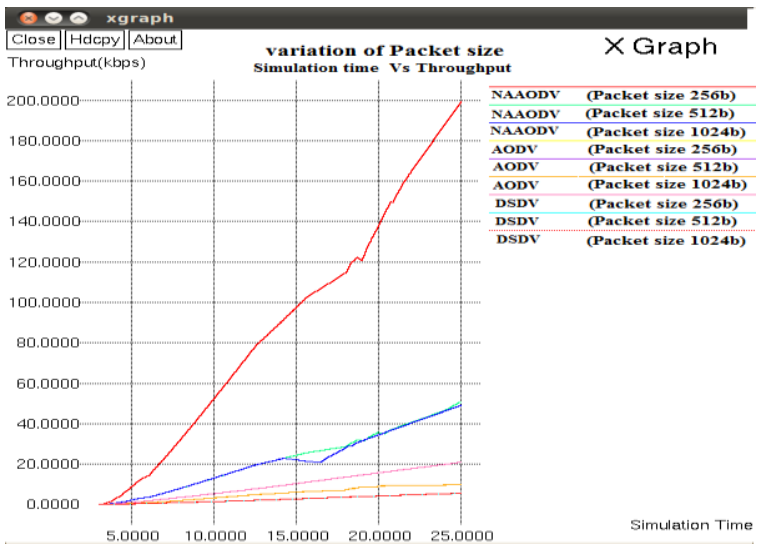

Figure 7: Throughput at variable packet size

\subsection{Impact of variable bit rate and packet size at Packet loss}

It has been identified that the packet loss in NAAODV is decreases at bit rates 100 and $500 \mathrm{~kb}$ which specifies the high reliability but NAAODV has larger end to end delay also random at higher data rate $1000 \mathrm{~kb}$ as shown in Figure 8. The number of dropped packets in AODV is moderate. For increased packet size from 256 b to 1024 bytes, NAAODV outperform regarding the delay comparison to AODV and DSDV as shown in Figure 9.

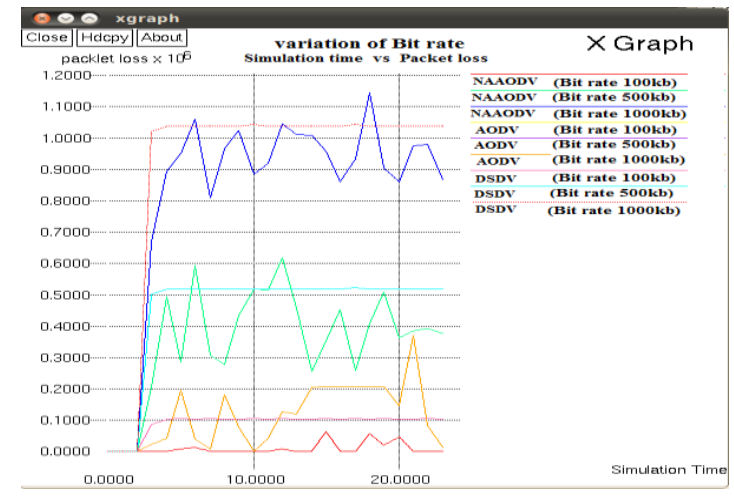

Figure 8: Packet loss at variable bit rate

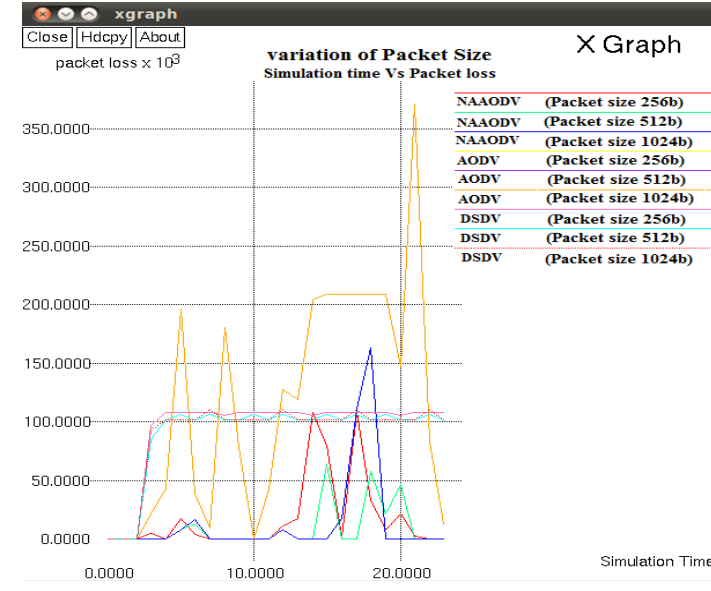

Figure 9: Packet loss at variable packet size

\subsection{Impact of variable bit rate and packet size at Delay}

NAAODV provides a less delay in most cases for variable packet size as shown in Figures 10 and 11. However, as the bit rate increases, NAAODV displays more delay then other two. From Figure 10,It is evident that there is a oscillatory behavior in end to end delay in NAAODV compared with AODV to DSDV at bit rat $1000 \mathrm{~kb}$.

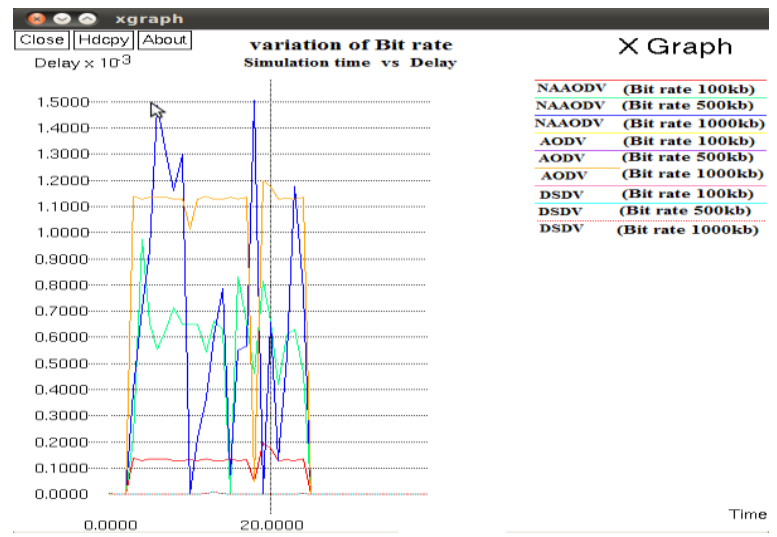

Figure 10: Delay at variable bit rate

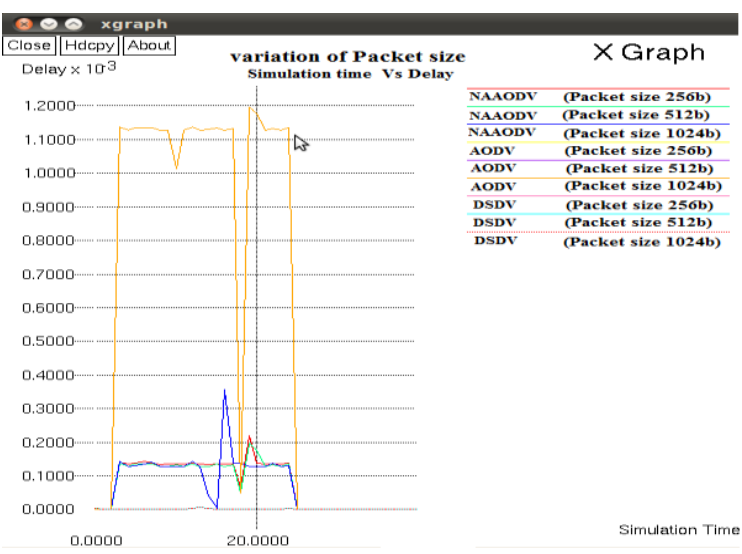

Figure 11: Delay at variable packet size 


\subsection{Impact of variable bit rate and packet size and node density at Packet delivery ratio.}

The NAAODV offers the highest increase in PDR than AODV and DSDV with increased packets size and node density. But still performance degrades in the proposed method for an increase in the data rate is observed from the bar chart given in Figure 12.

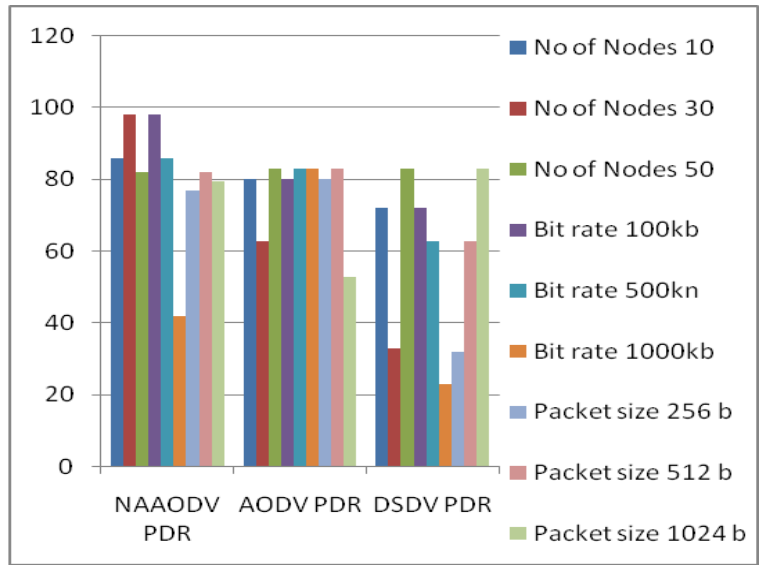

Figure 12: Packet Delivery Ratio at variable node density, bit rate and packet size

\section{CONCLUSION}

In the proposed NAAODV, a novel approach based on conventional AODV with different performance indices such that variable bit rate, packet size and node density. NAAODV has been investigated through simulations and contrasted against with similar other schemes to illustrate its viability for practical implementation. In terms of throughput, NAAODV shows better performance for data traffic received and throughput when increasing number of nodes and packet size except at higher bit rates because of rebroadcast optimization by priority assignment, less overhead and neighbor node energy information. AODV and DSDV shows poor performance, data loss is also significantly more when increased bit rate and packet size. The performance of NAAODV is beneficial in terms of throughput and packet loss compared to all other schemes.

\section{ACKNOWLEDGMENTS}

The authors thank the authorities of Annamalai University for offering the essential facilities in order to accomplish this piece of work.

\section{REFERENCES}

[1] Perkins CE, Royer EM "Ad-hoc on-demand distance vector routing". In the Proceedings of IEEE, WMCSA, pp. $90-100,19993$.

[2] Xin Ming Zhang, En Bo Wang, Jing Jing Xia, Dan Keun Sung "A Neighbor Coverage -Based Probabilistic
Rebroadcast for Reducing Routing Overhead in Mobile Ad Hoc Networks". Mobile Computing, IEEE Transactions on Volume: 12, Issue: 3 2013, Page(s): 424 $-433$.

[3] Q. Zhang and D. Agrawal. Dynamic probabilistic broadcasting in manets. Journal of parallel Distributed Computing, 65:220-233, 2005.

[4] The Marc Greis Tutorial: Tutorial for the Network Simulator "ns" Reformatted by Tae-yung Kim, On behalf of NSusers, January 10, 2006, from http://www.isi.edu/nsnam/ns/tutorial

[5] J. Broch, D.A. Maltz, D.B. Johnson, Y.-C. Hu, and J. Jetcheva, "A Performance Comparison of Multi-Hop Wireless Ad Hoc Network Routing Protocols," Proc. MobiCom, pp. 85-97, 1998.

[6] Hong Jiang, Garcia-Luna-Aceves, J.J. "Performance comparison of three routing protocols for ad hoc networks". Proceedings of the tenth International Conference on Computer communications and Networks, Oct 2001, pp. 547-554

[7] Larry C. Llewellyn, Kenneth M. Hopkinson, Member, IEEE, and Scott R. Graham, Member, IEEE, "Distributed Fault-Tolerant Quality of Wireless Networks", IEEE transactions on mobile computing, vol. 10, no. 2, February 2011.

[8] Matulya Bansal and Gautam Barua, "Performance Comparison of Two on Demand Routing Protocols for Mobile Ad hoc Networks" IEEE Personal Communication, 2002

[9] C. Zhu and S. CorsonC, "QoS Routing for Mobile Ad Hoc Networks", IEEE Infocom, 2002.

[10] Adib M.Monzer Habbal and Suhaidi Hassan, "Loss Detection and Recovery Techniques for TCP in Mobile Ad Hoc Network", SMIEEE in 2010 Second International Conference on Network Applications, Protocols and Services.

[11] N.Sumathi and Dr. Antony Selvadoss Thanamani, "Evaluation of Energy Efficient Reactive Routing Protocols in QoS Enabled Routing for MANETS", IJCA (0975 - 8887) Volume 14-No.2, January 2011.

[12] http://www.scribd.com/doc/55865371/Improving-QoSin-Mobile-Networks-Through-Effective-Routing.

[13] http://www.en.wikipedia.org/wiki/Quality_of_service

[14] K. Dridi, K. Djouani and B. Daachi, "Three Schedulinglevels algorithm for IEEE 802.11 QoS efficiency improvement", Proceedings of MoMM 2008, Linz, Austria.

[15] Roch A. Guerin and Ariel Orda, "QoS Routing in Networks with Inaccurate Information: Theory and Algorithms", IEEE/ACM TRANSACTIONS ON NETWORKING, VOL. 7, NO. 3, JUNE, 1999. 